\title{
Concepciones dinámicas y estáticas del infinito: procesos continuos y sus totalidades
}

\section{Dynamic and Static Conceptions of Infinity: Continuous Processes and their Totalities}

\author{
Diana Villabona Millán \\ Departamento de Matemática Educativa. \\ Cinvestav - IPN, Ciudad de México, México. \\ diana.villabona@gmail.com \\ Solange Roa Fuentes \\ Escuela de Matemáticas, Facultad de Ciencias, Universidad Industrial de Santander, Bucaramanga, Colombia. \\ sroa@matematicas.uis.edu.co \\ Asuman Oktaç \\ Departamento de Matemática Educativa, Cinvestav - IPN, Ciudad de México, México. \\ oktac@cinvestav.mx
}

RESUMEN - El estudio del infinito en matemática educativa desde una perspectiva cognitiva se ha centrado en analizar el infinito potencial y actual. A partir de la teoría APOE (Acción, Proceso, Objeto, Esquema), esta noción se ha explicado en términos de procesos iterativos infinitos y objetos trascendentes, respectivamente. En este artículo se presenta una descomposición genética, que sustenta el diseño de dos problemas relacionados con la recta tangente a una curva, en el que se toman en consideración los aspectos dinámicos y estáticos del infinito. A través de una entrevista realizada a un profesor universitario, se presenta evidencia de Totalidad, una posible nueva estructura que permite que el individuo conciba un Proceso como un todo, aunque no pueda aplicar Acciones sobre ella.

PALABRAS CLAVE: Infinito matemático; Teoría APOE; Totalidad; Procesos infinitos; Objetos trascendentes.

ABSTRACT • From a cognitive perspective, the study of infinity in mathematics education has focused on analyzing the potential and actual infinities. From the point of view of APOS (Action, Process, Object, Schema) theory, these notions have been explained in terms of iterative infinite processes and transcendent objects, respectively. In this article a genetic decomposition that supports the design of two problems related to the tangent line to a curve, where dynamic and static aspects have been taken into consideration, is presented. By means of an interview with a university instructor, evidence of Totality, a possible new structure that allows an individual to conceive a Process as a whole without being able to apply Actions to it is presented.

KEYWORDS: Mathematical infinity; APOS theory; Totality; Infinite processes; Transcendent objects

Recepción: abril 2020 • Aceptación: agosto 2021

Villabona, D., Roa Fuentes, S. y Oktaç, A. (2022). Concepciones dinámicas y estáticas del infinito: procesos continuos y sus totalidades Enseñanza de las Ciencias, 40(1), 179-197. https://doi.org/10.5565/rev/ensciencias.3277 


\section{LA PROBLEMÁTICA DEL INFINITO EN MATEMÁTICA EDUCATIVA}

El estudio epistemológico del infinito ha permitido develar la complejidad de su construcción cognitiva dada su naturaleza dual: potencial y actual. Dicha naturaleza es vista como un obstáculo de corte epistemológico (Artigue, 1995; Sierpinska, 1987) que es persistente y resistente a la formación matemática (Brousseau, 2002; Mena-Lorca, Mena-Lorca, Montoya-Delgadillo, Morales y Parraguez, 2015). Según Moreno y Waldegg (1991), los esquemas de respuesta de los estudiantes en situaciones relacionadas con el infinito se parecen a las respuestas de sujetos epistémicos (científicos que a través de la historia han logrado establecer estructuras de conocimiento general). Las intuiciones de los individuos sobre el infinito son netamente potenciales (Fischbein, 1987), ya que se desarrollan a partir de su interacción con el mundo real. Intuitivamente, el infinito se acepta en su forma dinámica, como un proceso que se repite sin fin (potencial). Estas intuiciones no funcionan cuando se trata de un infinito estático (actual); incluso llegan a convertirse en un obstáculo (Fischbein, 2001).

Dreyfus y Tsamir (2004) presentan evidencia de cómo los elementos del contexto, es decir, la situación matemática, entran en juego al construir procesos de abstracción; dichos procesos son el resultado de las acciones epistémicas: reconocer, edificar y construir. La construcción cognitiva del infinito está condicionada por el contexto en el cual se presenta; en este sentido, es posible identificar contextos dinámicos y contextos estáticos. Los contextos dinámicos contienen uno o más procesos explícitos en su enunciado. Por ejemplo, las paradojas de Zenón son contextos dinámicos, ya que en ellas pueden evidenciarse procesos que se llevan a cabo de forma iterativa (Villabona y Roa-Fuentes, 2016). Los contextos estáticos no evidencian procesos, sino objetos que surgen de procesos infinitos que ya han sido conceptualizados, por ejemplo, la paradoja del hotel de Hilbert presenta un hotel de infinitas habitaciones lleno (Roa-Fuentes y Oktaç, 2014). Otro criterio que se puede utilizar para clasificar los contextos del infinito se asocia a la representación de los elementos inmersos en un problema matemático, por ejemplo, geométrico o algebraico. Los contextos que se relacionan con objetos geométricos generan en los individuos argumentos asociados a la vida real (Sacristán, 1991). En contraste, un contexto algebraico puede propiciar el paso a una etapa interobjetal de acuerdo con la teoría piagetiana, que solo es posible cuando el individuo tiene la capacidad de ver conjuntos infinitos como entidades terminadas y puede establecer relaciones entre ellas (Moreno y Waldegg, 1991).

En este artículo utilizamos el término infinito matemático para referirnos a la noción relacionada con la construcción de procesos infinitos (infinito potencial) y sus estados al infinito (infinito actual), así como para diferenciarlo del uso del término infinito en la vida diaria. Estudios iniciales desde la perspectiva de la teoría APOE sobre la construcción cognitiva del infinito muestran que las concepciones asociadas a su construcción, en potencia y en acto, se relacionan con las estructuras de Proceso y Objeto, respectivamente (Weller, Brown, Dubinsky, McDonald y Stenger, 2004; Dubinsky, Weller, McDonald y Brown, 2005a; 2005b); de esta manera se muestra una interpretación alternativa de las dificultades de su aprendizaje a la ofrecida por la perspectiva de los obstáculos epistemológicos (en este artículo se diferencian las estructuras de la teoría escribiendo su primera letra en mayúscula). Estos Procesos tienen características de procesos iterativos infinitos y los Objetos son estructuras estáticas que trascienden de los Procesos que los generan, esto es, un Objeto que surge de la conceptualización de un proceso infinito no hereda las propiedades del Proceso (Brown, McDonald y Weller, 2010). Para Brown y otros (2010), "un proceso iterativo infinito es la aplicación infinita de una transformación sobre un objeto, ya sea cognitivo o físico, que envuelve uno a más parámetros que cambian con cada repetición» (p. 116).

Dubinsky, Weller y Arnon (2013) proponen una posible estructura mental denominada Totalidad, para explicar cómo los individuos comprenden la igualdad $0.999 \ldots=1$. Esta posible estructura debe ser evidenciada empíricamente en la construcción de distintos conceptos matemáticos, e incluso en 
contextos diferentes al de la expansión decimal de fracciones, para que pueda llegar a determinarse como una etapa de construcción de conocimiento en el marco de la teoría APOE.

Nuestro objetivo en este estudio es doble: por un lado, ofrecer evidencia de la posible estructura Totalidad en un contexto relacionado con el infinito, pero diferente a aquello que dio origen a las primeras observaciones (Dubinsky et al., 2013); y, por otro lado, avanzar y contribuir en la reflexión teórica sobre las estructuras mentales que conforman la teoría APOE.

\section{ACCIONES, PROCESOS, OBJETOS Y ESQUEMAS: LA TEORÍA APOE}

Desde la perspectiva de la teoría APOE, un individuo desarrolla su comprensión de conceptos matemáticos construyendo y utilizando estructuras mentales denominadas Acciones, Procesos, Objetos y Esquemas; dichas estructuras se consideran etapas en el aprendizaje de los conceptos, en línea con la teoría epistemológica de Piaget (Arnon, Dubinsky, Cottrill, Oktaç, Roa-Fuentes, Trigueros y Weller, 2014; Piaget y García, 1982).

La construcción de estructuras asociadas a un concepto particular se relaciona íntimamente con la concepción que un individuo alcanza sobre dicho concepto. Una concepción es algo «intrapersonal (es decir, la idea o comprensión del individuo)» (McDonald, Mathews y Strobel, 2000, p. 78); que «se desarrolla como resultado de una actividad reflexiva» (Arnon et al., 2014, p. 18). Cabe mencionar que dicho desarrollo no es lineal; hay un ir y venir entre diferentes etapas en la construcción del conocimiento matemático.

Las estructuras se construyen y se relacionan por medio de mecanismos tales como la interiorización, la encapsulación y la coordinación constituyendo marcos coherentes denominados Esquemas (Weller et al., 2004). En la figura 1 se presenta un Esquema elemental en el que se muestra la progresión $A \rightarrow P \rightarrow O \rightarrow E$ como una forma de relacionar las estructuras a partir de algunos mecanismos.

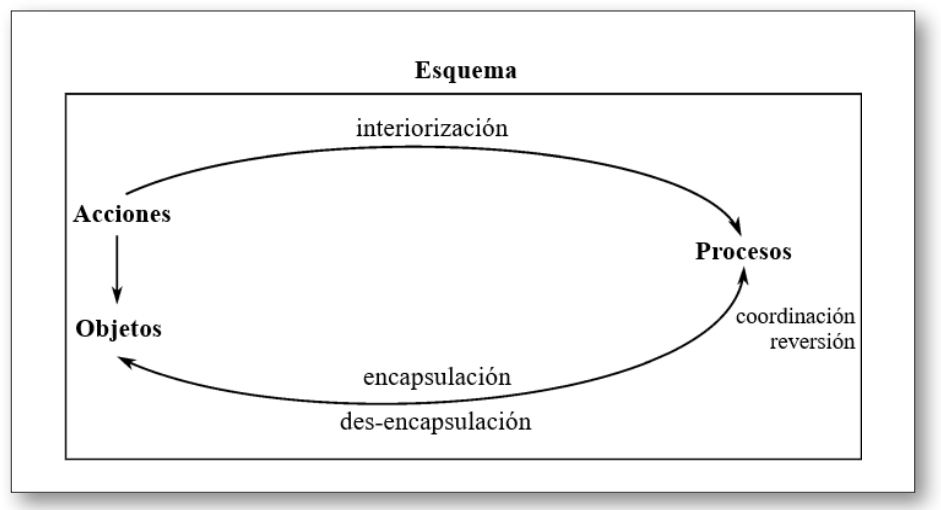

Fig. 1. Estructuras y mecanismos mentales para la construcción del conocimiento matemático (adaptado de Arnon et al., 2014)

Las Acciones son transformaciones que se realizan sobre un Objeto u Objetos previamente construido(s) por un individuo. Se caracterizan por ser el resultado de una instrucción directa y por la necesidad de ser llevadas a cabo explícitamente. Por ejemplo, una concepción Acción de función permite que un individuo transforme específicamente uno o más elementos de su dominio a partir de una expresión de la forma $y=f(x)$ (Breidenbach, Dubinsky, Hawks y Nichols, 1992). En este caso, la acción se realiza sobre los objetos que conforman el dominio de la función.

La construcción de la estructura Proceso depende del nivel de reflexión que se haya alcanzado durante la repetición de las Acciones. Dicha reflexión propicia el mecanismo de interiorización que le 
permite al individuo tener control interno de las Acciones que antes realizaba de forma externa. En el caso de las funciones, un individuo puede aceptar que todos los elementos del dominio de la función han sido transformados.

Cuando un individuo ha construido un Proceso y puede verlo como un todo sobre el cual puede realizar Acciones, se dice que encapsuló el Proceso en un Objeto. El mecanismo de encapsulación está motivado por la necesidad de realizar Acciones sobre el Proceso, lo que le permite pasar de una estructura dinámica (Proceso) a una estructura estática (Objeto). Un individuo con una concepción Objeto de función puede construir, por ejemplo, conjuntos de funciones y analizarlas como vectores de un espacio vectorial.

Otra forma de construir Procesos surge a partir del mecanismo de coordinación, donde el individuo necesita de dos o más Procesos que combina en uno, como en el caso de composición de funciones.

Uno de los principales objetivos de la teoría APOE es plantear modelos cognitivos de construcción de conceptos matemáticos; estos se denominan descomposiciones genéticas, las cuales se detallan en términos de estructuras y mecanismos mentales, y dibujan el camino que se puede seguir por etapas. La primera descomposición que se diseña para explicar la construcción de un concepto se conoce como preliminar, ya que no se basa en evidencia empírica, sino en un análisis teórico. Cuando mediante datos coleccionados y su análisis se puede confirmar la validez de este primer modelo, la descomposición genética se consolida. Si existe discrepancia entre los datos obtenidos y la descomposición preliminar, esta última sufre modificaciones para reflejar la evidencia y se llama descomposición genética refinada.

A continuación, se describen aspectos metodológicos que han guiado el curso de esta investigación. Estos corresponden a una adaptación del paradigma de investigación de la teoría APOE (Arnon et al., 2014).

\section{ASPECTOS METODOLÓGICOS}

En este artículo, a través de dos componentes del ciclo de investigación de APOE (Arnon et al., 2014), a saber, análisis teórico y recolección y análisis de datos, se dan a conocer los resultados de un estudio sobre la comprensión del infinito matemático. En la primera componente se presenta una descomposición genética de infinito que contempla la Totalidad. Este modelo guía el diseño de un instrumento compuesto por dos problemas relacionados con la recta tangente a una curva que no han sido estudiados previamente considerando la posible estructura Totalidad, aunque una descomposición genética del concepto de recta tangente enfocada a una descripción matemática se reporta en Orts, Llinares y Boigues (2016). Para la segunda componente, se analiza una entrevista realizada a un profesor universitario, quien logra construir el infinito como un todo, sin poder aplicar Acciones sobre él. Esto, como se explica con detalle en el desarrollo de este escrito, define a Totalidad.

Dado que la intención de este estudio es averiguar la existencia de la Totalidad como una posible estructura mental, el diseño de situaciones que pueden motivar tal construcción resulta primordial. En cuanto a participantes, trabajamos con trece personas con amplios conocimientos en matemáticas (profesores con experiencia en la enseñanza de matemáticas universitarias, estudiantes de posgrado en matemáticas y matemática educativa), con el fin de asegurar que no tuvieran dificultades con los conocimientos básicos requeridos para abordar dichas situaciones.

Debido al propósito de la investigación, así como el hecho de no contar con diseños anteriores enfocados en la Totalidad, el método que se siguió fue una adaptación del ciclo metodológico de la teoría APOE, donde hicimos uso de dos de los tres componentes, como se muestra en la figura 2 . Se llevaron a cabo varias iteraciones entre estos, y se mejoró el diseño de las situaciones en cada iteración, acercándonos cada vez más a situaciones que pueden evocar la manifestación de Totalidad. 


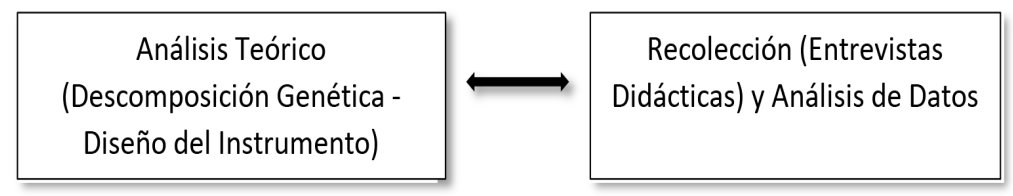

Fig. 2. Componentes que iteran en el diseño y la recolección de datos

La doble implicación señala una interacción continua entre los dos componentes. El diseño del instrumento se considera junto al análisis teórico, dado que el contexto tiene una relación estricta con la construcción del infinito matemático. Durante cuatro diferentes aplicaciones se refinó la descomposición genética preliminar, así como el instrumento de toma de datos, a partir de la evidencia empírica obtenida. En la última toma de datos, a partir de un caso particular, se evidenció la construcción de una concepción Totalidad, y ese caso es el que presentamos aquí junto a su análisis. En las secciones 4 y 5 ahondamos sobre dichos componentes.

\section{ANÁLISIS TEÓRICO: FUNDAMENTACIÓN TEÓRICA Y DESCOMPOSICIÓN GENÉTICA}

En la siguiente sección se presenta una síntesis de la componente de análisis teórico y, posteriormente, se propone la descomposición genética refinada, la misma que fue obtenida como resultado de las aplicaciones anteriores del instrumento y que guio la aplicación de los problemas que se utilizaron en la última toma de datos.

\section{Fundamentación teórica: el infinito matemático a través de la teoría APOE}

Un primer análisis de la construcción cognitiva del infinito matemático desde la perspectiva de APOE propone que el infinito potencial y actual corresponden a dos estructuras cognitivas diferentes de la misma noción, Proceso y Objeto, respectivamente (Dubinsky et al., 2005a; 2005b). Según Brown y otros (2010), la construcción de una concepción Proceso de infinito está íntimamente relacionada con un proceso de iteración sobre el conjunto de los números naturales, esto lo concluyen a la luz de la evidencia de los razonamientos de un grupo de estudiantes que abordan el siguiente problema:

$$
\text { Pruebe o refute: } \bigcup_{k=1}^{\infty} P(\{1,2,3, \ldots, k\})=P(\mathbb{N})
$$

donde $\mathbb{N}$ representa el conjunto de los números naturales y $P(\mathbb{N})$ representa el «conjunto potencia», esto es, el conjunto de todos los subconjuntos de un conjunto dado (Brown et al., 2010, p. 116).

El lado izquierdo de la afirmación propone la unión infinita de conjuntos finitos (forma dinámica) y el lado derecho el conjunto de partes de $\mathbb{N}$ (forma estática). Brown y otros (2010) muestran las dificultades de los estudiantes para analizar el lado derecho de la igualdad, ya que este no propone una forma dinámica de construcción. La construcción de una concepción Proceso de infinito requiere de la identificación de los procesos involucrados, su planteamiento como procesos iterativos y su posterior coordinación (Arnon et al., 2014; Villabona y Roa-Fuentes, 2016). En algunas situaciones pueden identificarse dos procesos infinitos que deben ser coordinados en uno, lo que Dubinsky, Weller, Stenger y Vidakovic (2008) denominan coordinaciones bi-dimensionales de procesos iterativos infinitos. 
Cada uno de los procesos puede tener naturaleza distinta (un proceso puede ser convergente, mientras el otro diverge), lo que hace que la coordinación sea particularmente difícil, dado que el individuo debe determinar cuál es la naturaleza del proceso que surge de la coordinación.

Para explicar el paso de una estructura dinámica (Proceso iterativo infinito) a una estática (Objeto trascendente), Roa-Fuentes y Oktaç (2014) proponen el mecanismo de completez, que permite a un individuo determinar las características estáticas de un proceso iterativo infinito. Este mecanismo fue inicialmente caracterizado en los razonamientos de algunos individuos mientras pensaban en procesos infinitos como terminados, característica que a la fecha en la que se culminó dicha investigación correspondía a la estructura Objeto y que en la actualidad ha permitido analizar la posible existencia de la estructura Totalidad.

Dada la influencia del contexto en la manera como los individuos abordan la construcción del infinito, en algunas investigaciones se ha propuesto una descomposición genética genérica de infinito (Roa-Fuentes y Oktaç, 2014; Villabona y Roa-Fuentes, 2016) que agrupa las características comunes en la construcción del infinito matemático en diferentes contextos para que sean adaptadas a cualquier contexto particular, ya sean dinámicos o estáticos. Dicha descomposición genética genérica toma en cuenta la construcción de procesos iterativos infinitos y el mecanismo de completez como aquel que permite ver dichos procesos como un todo.

La relación que existe entre el infinito dinámico y estático se debe analizar en términos del movimiento de un proceso infinito hacia su límite (Hauchart y Rouche, 1987). Una de las dificultades relacionadas con el concepto de límite es que se define como un proceso que no se completa (Cornu, 1992), es decir, una noción dinámica donde el límite nunca se alcanza (Cottrill et al., 1996), en vez de tomarse en cuenta su otra faceta: como un objeto sobre el cual se pueden realizar acciones. Sin embargo, solucionar múltiples situaciones requiere de una mirada estática del límite, lo que supone completar cognitivamente el Proceso en cuestión. Es por lo que Villabona y Roa-Fuentes (2016) proponen que, para que un individuo acepte como terminados procesos iterativos infinitos, son necesarias concepciones estáticas de conceptos como límite y convergencia, es decir, que el individuo pueda estructurar los Procesos asociados a estos conceptos como terminados; esto se relaciona con el mecanismo de completez.

Se han atribuido dos características principales a la concepción Objeto en la teoría APOE: (1) el individuo puede ver la Totalidad del Proceso que construyó, y (2) el individuo puede realizar Acciones sobre dicha Totalidad. Tradicionalmente, se ha considerado que (1) implica (2). Sin embargo, Dubinsky y otros (2013) demuestran que, en un contexto del infinito, estas dos características pueden corresponder a concepciones diferentes. Así surge la necesidad de proponer una estructura entre el Proceso y el Objeto, que denominaron Totalidad, y que permite al individuo estructurar Procesos como un todo. Un individuo con una concepción Totalidad puede imaginar que la transformación dada por el Proceso es llevada a cabo en una única aplicación, por lo que libera al Proceso de cualquier idea de temporalidad. Sin embargo, aunque empiece a concebir el Proceso como una entidad, como «algo terminado", no puede llevar a cabo Acciones sobre dicha entidad. Mientras que un individuo con una concepción Objeto puede aplicar Acciones sobre ese todo.

Las Acciones aplicadas sobre Objetos evolucionan gracias a la experiencia de cada individuo con situaciones en donde deba reflexionar sobre las propiedades del Objeto. La paradoja de la "Lámpara de Thomson» analizada por Wijeratne y Zazkis (2016, p. 128) permite profundizar en esta cuestión.

Piense en una lámpara con un botón de encendido/apagado. Suponga que el botón puede ser presionado en un instante de tiempo y que inicialmente la lámpara está apagada. Después de un minuto el botón es presionado y la lámpara se prende. Después de $1 / 2$ minuto el botón es presionado y la lámpara se apaga. $1 / 4$ de minuto después se presiona nuevamente el botón y la lámpara se prende, y se continúa así sucesivamente. 
Es decir, se presiona el botón de encendido/apagado de la lámpara cuando transcurre exactamente la mitad del intervalo de tiempo. Al final de dos minutos j̨la lámpara está encendida o apagada?

En esta paradoja se identifican dos procesos: la adición infinita de intervalos finitos de tiempo y la función binaria de apagar y prender la lámpara. Para Wijeratne y Zazkis (2016) resolver la paradoja requiere analizar lo que sucede en el intervalo de tiempo [2- 2,2$]$ con $0<\varepsilon<2$, donde la encapsulación no es esencial dado que no existe un Objeto que resulte del proceso de prender y apagar la lámpara. En contraste con esto, consideramos que solucionar la paradoja requiere de la aplicación de una Acción que permita al individuo imaginar si la lámpara está prendida o apagada justo cuando se alcanza la Totalidad de la adición de intervalos de tiempo, esto es, cuando pasan 2 minutos. Plantear la pregunta "¿después de dos minutos la lámpara está prendida o apagada?» sugiere la aplicación de una Acción sobre la Totalidad del Proceso relacionado con el paso del tiempo.

Wijeratne y Zazkis (2016) demuestran que algunos individuos evidencian inconsistencias al trabajar con esta paradoja; por ejemplo, al considerar que no se pueden alcanzar los 2 minutos y al mismo tiempo pensar en el estado (on/off) de la lámpara en ese instante. Esto surge porque los individuos no han podido construir la Totalidad del Proceso que se obtiene de la coordinación del Proceso dado por la adición infinita de intervalos de tiempo y el Proceso binario (on/off), por tanto, no pueden realizar la Acción que les permita dar una respuesta a la pregunta de la paradoja.

El diseño de un contexto donde sea viable estudiar procesos infinitos, sus totalidades y las acciones que puedan actuar sobre esas totalidades es uno de los principales objetivos de esta componente: el análisis teórico. En el siguiente apartado se presentan dos problemas que permiten analizar la estructuración de la Totalidad asociada al límite de un proceso continuo. Estos problemas consisten en contextos viables para analizar la construcción cognitiva del infinito -en potencia y en acto- porque requieren de la construcción de procesos infinitos y sus estados límite. Además, permiten analizar por primera vez la construcción del infinito en términos de la teoría APOE mediante procesos continuos y los objetos que surgen de ellos, así como estudiar la posibilidad de que a pesar de que un individuo construya la Totalidad de un proceso infinito no pueda llevar a cabo Acciones sobre ella. Cabe mencionar que los estudios anteriores del infinito fundamentados teóricamente en APOE se centran en la estructuración de procesos iterativos infinitos y sus Objetos trascendentes.

\section{Descomposición genética refinada del problema de la pendiente de la recta tangente a una curva en un punto}

Esta descomposición genética se obtuvo tras llevar a cabo diversas iteraciones del ciclo de investigación y surgió a partir de una adaptación teórica a la descomposición genética genérica de infinito (Villabona y Roa-Fuentes, 2016). La adaptación toma en cuenta la construcción de procesos infinitos continuos (construcción de un infinito no numerable) a partir de un pensamiento «iterativo", la estructura Totalidad como una posible etapa en la construcción del infinito matemático y la completez como el mecanismo que da paso a la Totalidad. Además, se propone la encapsulación como el mecanismo que permite realizar Acciones sobre las Totalidades de procesos infinitos. A continuación, presentamos el problema de la recta tangente.

i. Encuentre la pendiente de la recta tangente a la curva $f$ en el punto $P$ (este es un problema muy conocido en el cálculo de una variable; se denomina problema de la recta tangente). Supongamos que $f$ es continua en un intervalo que contiene a $x$ (ver figura 3 ). 


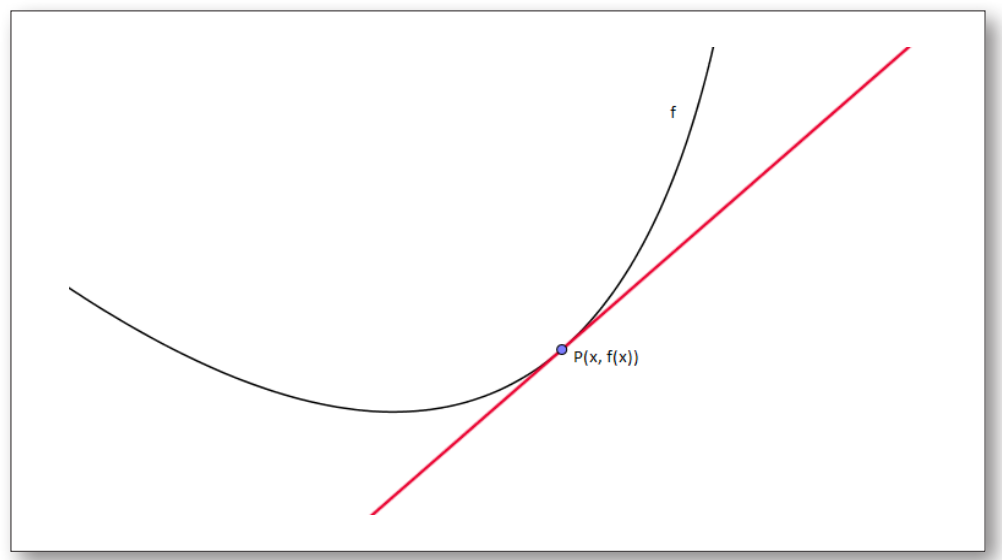

Fig. 3. Problema de la recta tangente

Una forma de abordar este problema es construir un proceso infinito y continuo de rectas secantes $\overleftrightarrow{P Q}$ ( $Q$ es un punto cualquiera sobre $f$ ) que, en el estado al límite cuando $Q \rightarrow P$, converge a la recta tangente a $f$ en $P$. Esta no es la única manera de afrontar matemáticamente el problema de la pendiente de la recta tangente. Sin embargo, es un método común en cursos de cálculo diferencial. Aquí, el Proceso asociado a este acercamiento infinito se denomina $P_{12}$; este Proceso surge de la coordinación de dos Procesos: el Proceso $P_{1}$ relacionado con la elección de puntos $Q$ sobre $f$ cada vez más cercanos al punto $P$ y el Proceso $P_{2}$ asociado a la construcción de rectas secantes a una curva. Las rectas secantes se definen a partir de los puntos $P$ y $Q$, esto hace que $P_{1}$ y $P_{2}$ se construyan y coordinen a la par a través de Acciones guiadas por la implicación: «una posición particular para el punto $Q \Rightarrow$ una única recta secante $\overleftrightarrow{P Q}$ ». La aplicación y reflexión sobre estas Acciones permite estructurar el Proceso $P_{12}$ (ver figura 4$)$.

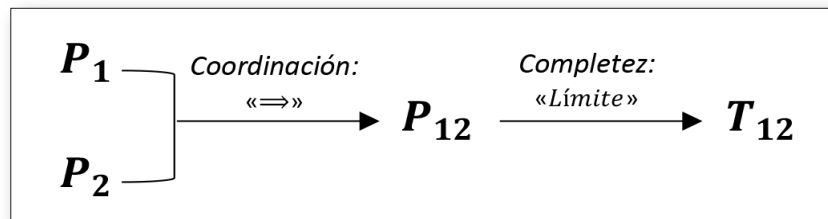

Fig. 4. Construcción de $P_{12}$ y $T_{12}$

El proceso $P_{12}$ tiene una naturaleza convergente que surge de la intención de acercar $Q$ a $P$ tanto como sea posible y esto se logra a través del concepto de límite, que permite generar una «sucesión» infinita y continua de rectas secantes $\overleftrightarrow{P Q}$ que converge a la recta tangente a $f$ en $P$. Cuando el individuo acepta que en la situación límite la pendiente de la recta $\overleftrightarrow{P Q}$ es la pendiente de la recta tangente, se considera que ha desarrollado el mecanismo de completez para construir la Totalidad $T_{12}$ del Proceso $P_{12}$. Un individuo con concepciones dinámicas de límite, en cambio, puede concluir que lo que encuentra es una buena aproximación de la recta tangente.

El problema anterior se soluciona cuando el individuo puede construir una concepción Totalidad de infinito $\left(T_{12}\right)$. Para saber si puede realizar Acciones sobre $T_{12}$, se diseńa y estudia el siguiente problema.

ii. En la siguiente construcción, determine la relación y si es posible la medida de los ángulos $A, B$, $C$ y $D$ en la situación límite cuando $Q \rightarrow P$. Las rectas $g$ y $h$ son paralelas entre sí y tangentes 
a la circunferencia $\tau$ (la recta $h$ es tangente a $\tau$ en el punto $P$ y $Q$ es un punto cualquiera sobre la circunferencia $\tau$ ) (ver figura 5).

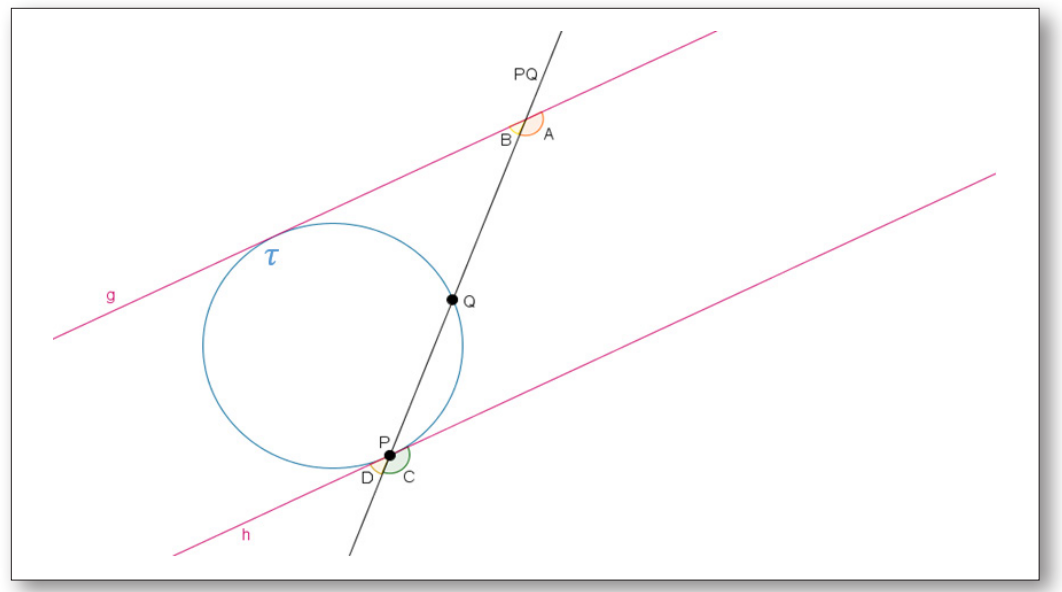

Fig. 5. Situación contexto relacionado con el contexto de las rectas tangentes

Resolver este problema requiere la aplicación de una Acción específica sobre $T_{12}$, ya que se necesita determinar las implicaciones que pueden tener las propiedades de dicha Totalidad. Este tipo de Acciones pueden ser analizadas haciendo uso de los elementos de la teoría APOE. Asiala y otros (1996) plantean que en ocasiones la realización de Acciones sobre Objetos puede requerir que el individuo vuelva al Proceso para determinar las propiedades de dicho Proceso y establecer cómo llevar a cabo la Acción. La realización de esta Acción en particular requiere que el individuo regrese sobre los Procesos que construyó en el problema $i$., los coordine con un nuevo Proceso (dado por el problema ii.) y obtenga un Proceso único que posteriormente totalizará. Si logra determinar las nuevas propiedades que tiene esta Totalidad, se dice que ha construido el Objeto trascendente, ya que determinar las propiedades de la Totalidad significa, en términos de la teoría APOE, realizar Acciones sobre esta.

De los Procesos $P_{1}$ (establecimiento de puntos $Q$ cada vez más cercanos a $P$ ) y $P_{2}$ (construcción de rectas secantes) se define un Proceso $P_{12}$ (generación de rectas secantes $\overleftrightarrow{P Q}$ cuando $Q \rightarrow P$ ). En el problema ii., las rectas secantes intersecan las rectas paralelas $g$ y $h$ que generan un Proceso $P_{123}$ (medidas de los ángulos $A, B, C$, y $D$ cuando $Q \rightarrow P$ ). Solucionar este problema requiere la construcción de $P_{123}$ a través de la coordinación de $P_{1}, P_{2}$ y $P_{3}$, donde $P_{3}$ es el Proceso asociado a la medida de los ángulos $A, B, C$, y $D$. Estos Procesos se coordinan mediante dos implicaciones: «una posición dada para el punto $Q \Rightarrow$ una única recta secante $\overleftrightarrow{P Q} \Rightarrow$ medidas particulares para los ángulos $A, B, C, \mathrm{y}$ D» (ver figura 6).

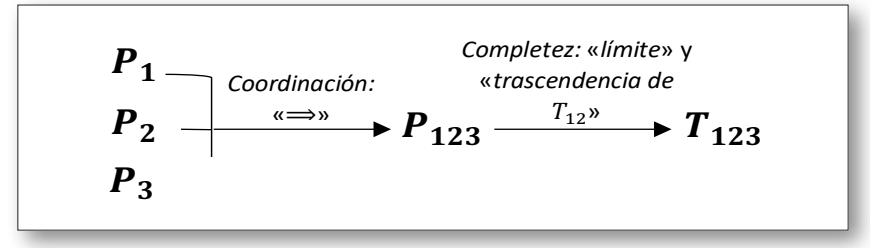

Fig. 6. Construcción de $P_{123}$ y $T_{123}$

$T_{12}$ (Totalidad en el problema $i$.) establece la naturaleza del Objeto que trasciende del Proceso $P_{123}$, lo que permite construir la Totalidad de este Proceso $\left(T_{123}\right)$. La construcción de $T_{123}$ se evidencia cuan- 
do el individuo concluye que la recta $\overleftrightarrow{P Q}$ se convierte en la recta $h y$, por tanto, no interseca la recta $g$, ya que es paralela a esta. De esta manera, el individuo puede pensar en la medida de los ángulos en términos actuales estableciendo que $A$ y $B$ no están definidos y $C$ y $D$ miden $180^{\circ}$ y $0^{\circ}$, respectivamente.

\section{RECOLECCIÓN Y ANÁLISIS DE DATOS}

En el desarrollo de este componente se aplicaron entrevistas de corte didáctico, donde se pueden proporcionar pistas al entrevistado con el fin de favorecer la construcción de conceptos durante su desarrollo (Oktaç, 2019). En este sentido, el objetivo del instrumento no solo fue determinar las posibles concepciones involucradas en la construcción del infinito en el contexto planteado, sino también poner a prueba la solidez de dichas concepciones a través de diversos momentos de conflicto generados por la interacción con la entrevistadora (primera autora de este artículo).

Los datos recolectados fueron analizados por las tres autoras de este artículo, de manera individual, y posteriormente comparados, hasta llegar a un acuerdo. A continuación, se presenta el análisis de la última toma de datos.

\section{Miguel y una concepción Totalidad de infinito}

Miguel es un profesor universitario que dirige cursos de geometría euclidiana y cálculo diferencial. Miguel enfrenta el contexto (problema i.) evidenciando concepciones estáticas del proceso infinito que surge como una coordinación de dos Procesos: uno asociado al proceso que se lleva a cabo en el dominio y que permite establecer un punto $Q$ sobre $f$ (Proceso $P_{1}$ ) y otro relacionado con la construcción de rectas secantes a una curva (Proceso $\left.P_{2}\right)$. Define el punto $Q(x+a, f(x+a))$ sobre la curva $f$ y construye la recta $\overleftrightarrow{P Q}$ (ver figura 7).

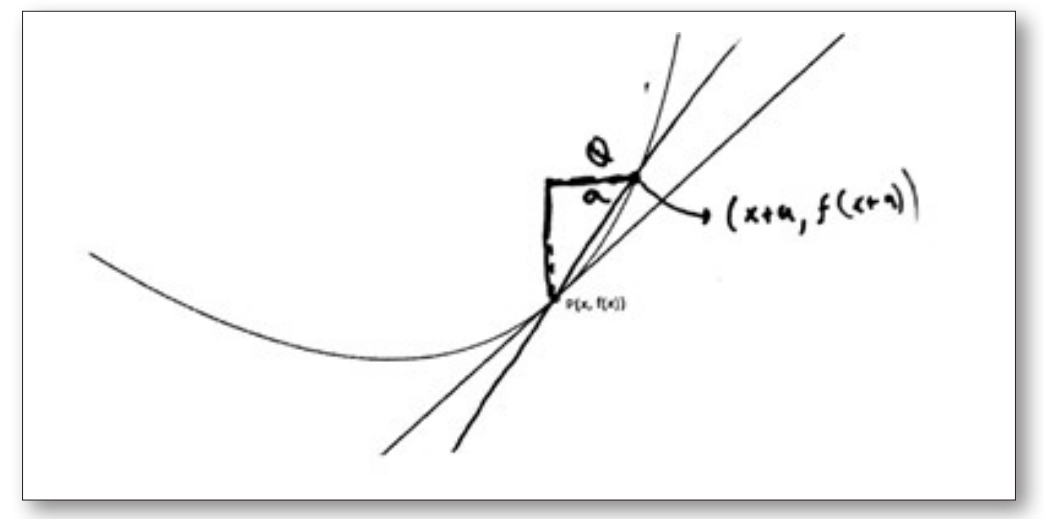

Fig. 7. Construcción de la recta PQ por parte de Miguel

Posteriormente determina la pendiente de la recta $\overleftrightarrow{P Q}$ (ver figura 8):

$$
m=\frac{f(x+4)-f(x)}{a}
$$

Fig. 8. Pendiente de la recta secante según Miguel 
Miguel plantea que $a$ (distancia que separa a $Q$ de $P$ ) debe hacerse tan pequeña como sea posible para que las rectas secantes se aproximen a la recta tangente a $f$ en $P$. Eso sucede porque Miguel ha construido a $P_{12}$ con antelación:

Miguel: Y... Y si $a$ se hace más pequeño entonces este punto [señalando a $Q$ ] se vuelve más cercano a $P$. Entonces esta recta [señalando la recta formada por los puntos $P$ y $Q$ ] se parece más a esta [señalando la recta tangente a la curva $f$ en $P$ ].

Miguel muestra una concepción Proceso planteando un proceso continuo de rectas secantes generadas por un punto $Q$ móvil a lo largo de $f$; estas rectas le permiten acercarse a la recta tangente tanto como quiere, haciendo uso del concepto de límite. Miguel puede construir este proceso como una totalidad:

Miguel: Eh... Cuando haya terminado de acercarme totalmente, serán la misma [refiriéndose a la recta pendiente y a la recta secante]. Pero me voy a acercar al estilo de Aquiles, reflexionando [risas] para que no llegue a dejar de ser recta jamás. Esta es la pendiente y la voy a llamar $p, p$ minúscula [escribe] (ver figura 9):

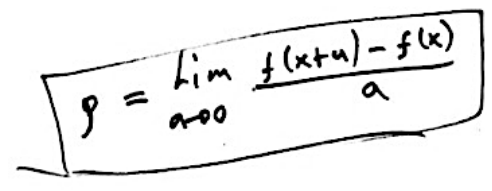

Fig. 9. Pendiente de la recta tangente según Miguel

Miguel evidencia que ha construido $T_{12}$ al manifestar: «cuando haya terminado de acercarme totalmente, serán la misma». Sin embargo, la trascendencia que sufre la recta $\overleftrightarrow{P Q}$ en el estado límite es algo que le preocupa; entonces expresa: «me acercaré al estilo de Aquiles (haciendo referencia a la paradoja de Zenón), para que no deje de ser recta jamás». Teniendo en cuenta esto se le pregunta qué pasa cuando $a=0$.

Miguel: Es que hay un detalle y es que decir que $a$ tiende a cero no es lo mismo que decir que $a$ es cero [silencio]. Sin embargo, no es una cantidad tampoco [silencio]... No es un número mayor que cero en ese momento, porque si lo fuera hay distancias más pequeñas ahí.

Miguel menciona que $a$ no puede ser igual a cero porque de ser así la expresión que escribió, y que permite calcular la pendiente de la recta tangente, se indetermina. Pero además plantea que $a$ tampoco puede ser una cantidad mayor que cero porque siempre podría escoger un $a$ más pequeño. Según el razonamiento de Miguel, $P_{1}$ no llega a totalizarse; es decir, evidencia concepciones dinámicas de lo que significa que una variable tienda a un valor real. Este tipo de concepciones son cómodas para él, ya que pensar en la tendencia como un proceso inacabado evita que la recta $\overleftrightarrow{P Q}$ deje de existir. Ahora se busca determinar qué pasa con el proceso que genera rectas tangentes en el límite $\left(P_{12}\right)$ y si Miguel puede estructurar su totalidad. Por tanto, se le pregunta si la expresión que encontró (ver figura 9) corresponde a la pendiente de la recta tangente o es una buena aproximación.

Miguel: No, es exactamente la pendiente. Lo que pasa es que mientras $a$ no sea cero, no será la pendiente. Sin embargo, cuando calculo el límite, $a$ no es una cantidad mayor que cero, a la vez que no es cero. Es raro... [Silencio prolongado] Ummm... Realmente este número [señalando el límite] no va a existir cuando $a$ sea cero, no es cero, pero tampoco es una cantidad mayor que cero. 
Miguel evidencia la totalidad $T_{12}$, aunque no estructura el proceso $P_{1}$ como un todo. Las concepciones estáticas de límite que manifiesta son un elemento importante que se relaciona con el mecanismo que le permite ver procesos infinitos como un todo. Esto indica que el proceso de tendencia de una variable a un valor real $\left(P_{1}\right)$, aunque es un proceso necesario para la construcción de Totalidad del proceso del límite, no requiere ser totalizado. En los casos en que existen discontinuidades, la idea de totalizar estos Procesos, aunque sea en un sentido metafórico, puede resultar compleja matemáticamente. En este caso, la recta $\overleftrightarrow{P Q}$ no está definida si se asume la distancia entre $Q$ y $P$ como cero.

Ahora, fue necesario determinar si Miguel puede llevar a cabo la acción propuesta en el problema ii.; al abordar dicho problema propone:

Miguel: Entonces, el punto... A medida que $Q$ se acerca, este ángulo decrece [seńalando el ángulo $D$ ] y entre más cerca esté de $P$ más cerca a cero estará este [señalando el ángulo $D$ ]. Entonces, en efecto, cuando $Q$ esté en $P$, cuando tienda a $P$, que no es que esté en $P, D$ tiende a cero. Entonces [concluye escribiendo] (ver figura 10):

\section{Si $Q \rightarrow P \quad P \rightarrow O$}

Fig. 10. Análisis de Miguel sobre el comportamiento del ángulo $\mathrm{D}$ cuando $\mathrm{Q} \rightarrow \mathrm{P}$

En el problema $i$. Miguel había concluido que en la situación al límite cuando $Q \rightarrow P$, la recta $\overleftrightarrow{P Q}$ sería la recta tangente $\left(T_{12}\right)$, sin embargo, ahora ignora el proceso generado por las rectas $\overleftrightarrow{P Q}$ y su estado al límite. Esto muestra que está coordinando $P_{1}$ (asociado a la tendencia de $Q$ a $P$ ) con $P_{3}$ (asociado a la medida de los ángulos $A, B, C$ y $D$ ). La entrevistadora pregunta de forma explícita si la medida del ángulo $D$ tiende a cero o es cero, a lo que Miguel responde:

Miguel: Tiende... Bueno, vuelvo al dilema de ahorita. Esto es una cantidad infinitamente... Digamos la distancia que hay entre $Q$ y $P$ es una cantidad infinitamente pequeña y esta también [señalando $D$ ]. Podríamos filosofar sobre si es cero o no es cero, lo que sí sé es que no es una cantidad mayor que cero, no es una cantidad finita que pueda dividir, es indivisible. Al poderla dividir estoy diciendo que puedo estar más cerca... Entonces [termina de escribir] (ver figura 11):

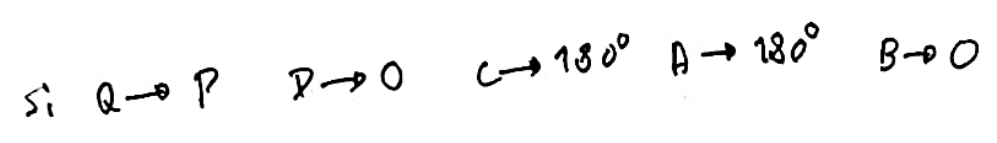

Fig. 11. Comportamiento de los ángulos cuando $\mathrm{Q} \rightarrow \mathrm{P}$ según Miguel

Miguel construye un proceso $P_{13}$ coordinando $P_{1}$ con $P_{3}$, excluyendo el Proceso asociado a la construcción de rectas secantes. Aunque el problema pide determinar la medida de los ángulos en la situación al límite cuando $Q \rightarrow P$, el proceso $P_{3}$ se define gracias a las rectas $\overleftrightarrow{P Q}$; no pensar en las rectas secantes y su convergencia es problemático. $P_{13}$ hereda la naturaleza dinámica del Proceso dado por $Q \rightarrow P$. A continuación, la entrevistadora solicita a Miguel que explique su solución.

Miguel: Ehhh... Este ángulo $B$ es congruente con este ángulo acá [señalando el ángulo opuesto al ángulo $D$ ] y estos dos [señalando el ángulo $D$ y su opuesto] son opuestos por el vértice y por lo tanto $B$ y $D$ son congruentes. El ángulo $A$ y el ángulo $C$ son correspondientes porque estas rectas son paralelas [señalando las rectas $\mathrm{g}$ y h] y por lo tanto puedo decir que [escribe] (ver figura 12): 


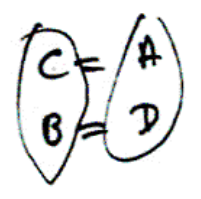

Fig. 12. Relación entre los ángulos según Miguel

Entonces con estos dos [resaltando los ángulos $C$ y $B$ ] puedo encontrar la magnitud de estos dos [resaltando los ángulos $A$ y $D]$. Y haciendo que $Q$ se acerque... Que tienda a $P$, que no es lo mismo decir que se acerque, $D$ tiende a cero y $C$ tiende a ciento ochenta. Esa es una forma, la otra forma es imaginar esta recta [señalando la recta $P Q$ ] que va cortando cada vez más allá [señalando con su dedo los puntos sobre la recta $g$ a la derecha de la hoja], entonces este ángulo [señalando el ángulo $C$ ] va a ser $180^{\circ}$ y este [señalando el ángulo $D$ ] va a ser $0^{\circ}$. Quedarían paralelas.

Miguel plantea dos posibles soluciones al problema, aunque resultan contradictorias. En la primera (solución dinámica) explica su postura inicial en la que no toma en cuenta el proceso de generación de rectas $\overleftrightarrow{P Q}$ que se da cuando $Q$ tiende a $P$ (asociado a $P_{12}$ ). Reflexiona sobre los ángulos sin pensar en las rectas que los definen, ni en lo que pasa con esas rectas en la situación límite; nuevamente, Miguel coordina $P_{1}$ con $P_{3}$. Para Miguel, el proceso de acercamiento de $Q$ a $P$ no se completa, es un proceso inacabado; el punto $Q$ jamás llegará a estar en $P$ y esto hace que el proceso de los ángulos $\left(P_{13}\right)$ se convierta también en un proceso dinámico que tampoco llega a completarse (ver figura 13).

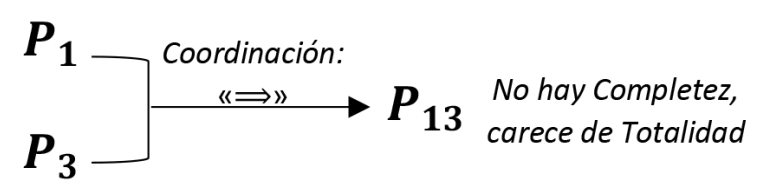

Fig. 13. Análisis de la solución dinámica de Miguel

En la segunda (solución estática), Miguel toma en cuenta el proceso de generación de rectas secantes, pero no lo relaciona explícitamente con el proceso de acercamiento de $Q$ a $P$ (por tanto, no llega al Proceso $P_{12}$ ), sino con un proceso $\left(P_{2}^{*}\right)$ determinado por el punto de intersección de la recta $\overleftrightarrow{P Q}$ con la recta $g$ y cómo este punto se extiende infinitamente a lo largo de $g$. De la solución del problema $i$. Miguel sabe que el proceso de rectas secantes trasciende y se convierte en la recta tangente gracias al límite y, por tanto, supone que lo mismo ocurrirá con la medida de los ángulos. Este análisis que se refiere como una solución estática solo es posible porque Miguel deja de lado el proceso de acercamiento de $Q$ a $P$, que es precisamente el proceso que al ser visto como terminado hace que la recta $\overleftrightarrow{P Q}$ resulte indeterminada (figura 14).

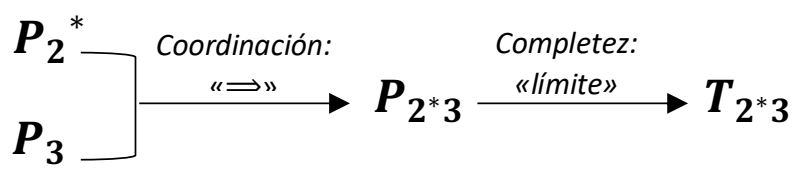

Fig. 14. Análisis de la solución estática de Miguel

Para descartar que el planteamiento de estas dos soluciones se deba a que Miguel no ha construido realmente $T_{12}$, se cuestiona la solución que ofreció en el problema $i$. 
E: En este límite [señalando la solución del problema i.] me habías dicho que ese límite no era una buena aproximación, sino que era la pendiente...

Miguel: Es la pendiente.

Miguel evidencia de nuevo y de forma contundente un pensamiento estático relacionado con el problema $i$. La entrevistadora confronta la naturaleza actual de las concepciones estáticas del límite en la solución del problema $i$. con la naturaleza potencial de la solución dinámica del problema ii. para averiguar si Miguel puede pensar exclusivamente en términos de la Totalidad de Procesos y no a través de Procesos inacabados. Miguel explica la solución del problema i. de la siguiente manera:

Miguel: Si lo veo como un proceso de: «me acerco y cada vez más cerca y cada vez más cerca», siempre voy a tener una diferencia con la pendiente de la recta tangente pero una vez que haya acabado el proceso, que es cuando digo « $a$ tiende a cero», que no es hacer a $a$, cero; es esa la pendiente.

Las concepciones dinámicas del proceso de tendencia están muy marcadas en el pensamiento de Miguel. Su defensa de la solución dinámica que presentó del problema ii. se centró en la imposibilidad de que $Q$ esté sobre $P$ y cómo esto hace que el ángulo $D$ nunca llegue a medir cero grados.

E: O sea que acá en esta situación [señalando el problema ii.] que es prácticamente un límite, lo que reflexionabas, cuando $Q$ tiende a $P$, ¿ $D$ no va a llegar a ser cero nunca?

Miguel: Mientras que $Q$ no esté en $P, D$ no es cero. Pero si tiende a $P, D$ tiende a cero [silencio prolongado]. Si $Q$ tiende a $P, D$ tiende a cero. Que no es lo mismo que decir que $Q$ es el punto $D$.

Las dos soluciones que plantea Miguel corresponden cognitivamente a dos coordinaciones separadas de los tres Procesos que debía coordinar. Los Procesos que surgen de la coordinación de $P_{1}$ con $P_{3}$ y de $P_{2}^{*}$ con $P_{3}$, por separado, generan dos soluciones contradictorias. $P_{1}$ no es un Proceso que por sí solo ha sido totalizado. El Proceso que ha sido totalizado es el que involucra la idea de límite, es decir el Proceso $P_{12}$. Esto hace que Miguel proponga una solución dinámica a la situación, donde la variación de los ángulos termina. Por otro lado, el proceso de generación de rectas secantes que Miguel usa no toma en cuenta de forma explícita el proceso de acercamiento de $Q$ a $P$ (proceso que permite definir todos los procesos que se llevan a cabo en los dos problemas del instrumento), sino que elige pensar en el punto de intersección de las rectas $\overleftrightarrow{P Q}$ y $g\left(P_{2}^{*}\right)$ ( La naturaleza del proceso de tendencia de $Q$ a $P$ le resulta problemática para Miguel; es casi como aceptar que $Q$ desaparece en el estado al límite. Para él es más fácil pensar que el punto de intersección de las rectas $\overleftrightarrow{P Q}$ y $g$ está en el infinito, ya que esto no interfiere con la existencia de la recta $\overleftrightarrow{P Q}$.

El dinamismo asociado a $P_{1}$ lleva a Miguel a proponer dos soluciones alternas y contradictorias de una misma situación, sin que esto le genere inconsistencias. Miguel puede pensar en el Proceso como un todo en la situación $i$., pero no puede solucionar satisfactoriamente la situación ii., no logra realizar la Acción propuesta para que actúe sobre $T_{12}$. Esto evidencia que Miguel no ha construido el Objeto que surge de este Proceso y, por tanto, tiene una concepción Totalidad de infinito.

\section{CONCLUSIONES}

A través del análisis presentado se postula por primera vez y en un contexto diferente al usado por Dubinsky y otros (2013), que a pesar de que un individuo pueda ver un proceso infinito como un todo, esto no implica que pueda realizar acciones sobre él. Se valida la progresión $A \rightarrow P \rightarrow T$ para la comprensión del infinito matemático en los contextos estudiados. A través de los razonamientos de un individuo, se evidencia que es posible construir procesos infinitos como entidades, sin que esto impli- 
que que se puede actuar sobre estas. Otro resultado de este estudio concierne al tránsito entre dichas estructuras; esto es, el mecanismo que permite ver procesos como terminados y el que permite actuar sobre dichos procesos no son el mismo. Tradicionalmente, ambas características estaban asociadas al mecanismo de encapsulación y la estructura Objeto, lo que para la comprensión del infinito resulta insuficiente.

Se propone teóricamente al mecanismo de completez como aquel que permite el paso de una concepción Proceso a una concepción Totalidad; y al mecanismo de Encapsulación como el que permite el desarrollo de una concepción Objeto. Las evidencias empíricas aquí presentadas respaldan la elección de completez como el mecanismo que permite el paso del Proceso a la estructura Totalidad, en el caso del infinito (ver figura 15). Cabe reiterar que para que la Totalidad se incluya dentro de las etapas de construcción de conocimiento, faltaría evidenciarla en conceptos diferentes al infinito matemático.

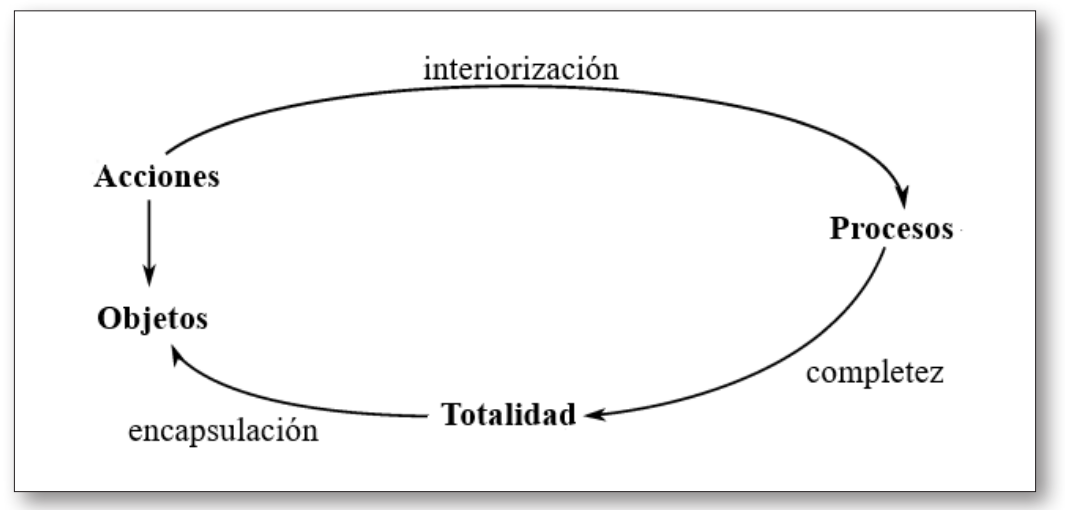

Fig. 15. Teoría APOE con la posible estructura Totalidad

En este estudio se propone un modelo viable de construcción del infinito en el contexto expuesto, que incluye la Totalidad. El proceso infinito continuo que Miguel evidencia trae consigo la idea de límite, clave para determinar la concepción que posee sobre el infinito en este contexto. Miguel propone que la situación al límite del proceso que genera pendientes de rectas secantes está directamente relacionada con la pendiente de la recta tangente. Esclarecer esta relación es importante, ya que puede hacer la diferencia entre una estructura dinámica de infinito y una estática. Con esto se afirma que, aunque un individuo plantee explícitamente que:

$$
m_{\text {tan }}=\lim _{Q \rightarrow P} m_{P Q}
$$

esto no implica que vea el proceso infinito continuo como un todo y que acepte que se obtiene la pendiente de la recta tangente al analizar el límite. En los procesos infinitos que son analizados a través de una situación al límite, la comprensión que tenga el individuo sobre este concepto hace la diferencia entre ver un proceso infinito como inacabado o verlo como un todo (infinito potencial e infinito actual). Por tanto, las concepciones dinámicas y estáticas del límite se relacionan con las concepciones que tenga el individuo del conjunto infinito sobre el cual el límite se defina. En este caso en particular, la situación al límite no se lleva a cabo sobre una sucesión o serie, sino sobre una función definida en un intervalo real. Lo anterior puede ayudar a enriquecer lo propuesto por Villabona y Roa-Fuentes (2016) sobre el mecanismo de completez. A continuación, se propone una nueva caracterización de este mecanismo que permite la construcción de la Totalidad de procesos infinitos iterativos y continuos. 
Completez: Es un mecanismo mental que le permite a un individuo imaginar las características que tiene la Totalidad de un Proceso infinito. El establecimiento de este mecanismo requiere de una construcción consciente de elementos fundamentales relacionados con la teoría de conjuntos: la relación entre un conjunto infinito y sus subconjuntos propios, los conceptos de cardinal y ordinal y la construcción de conjuntos infinitos con diferentes cardinalidades, específicamente, la construcción de concepciones Objeto del conjunto de los números naturales (para las totalidades de procesos iterativos infinitos) y del conjunto de los números reales (para las totalidades de procesos continuos). Estos elementos permiten la construcción de una concepción estática de infinito que puede no heredar las propiedades de los estados finitos del proceso infinito asociado.

La concepción que tiene Miguel del límite le permitió ver el proceso infinito como un todo, aceptando que el límite de las pendientes de las rectas secantes es la pendiente de la recta tangente, no una aproximación. Las Acciones que se aplican sobre Totalidades pueden ser altamente complejas. En la Acción que se espera en el problema ii., el individuo establece una propiedad de la Totalidad construida previamente: determinar la medida de los ángulos generados por la intersección entre la recta secante y otra recta, en el estado al límite. La imposibilidad de coordinar los tres procesos involucrados en esta Acción y posteriormente desarrollar concepciones estáticas de este hace que Miguel no logre determinar el estado actual de los ángulos cuando $Q$ está infinitamente cerca de $P$. El mecanismo de encapsulación en esta situación exige que el individuo asuma la Totalidad como una entidad terminada y no a partir del dinamismo que la generó. Además, dicho mecanismo debe permitirle reconocer que las propiedades de los procesos infinitos no son necesariamente heredadas del objeto que se obtiene en el estado al límite. El individuo debe desarrollar la capacidad de imaginar la naturaleza del Objeto, es decir, debe determinar su trascendencia (Brown et al., 2010). Para esto se hace necesario que enfrente situaciones novedosas donde reflexione sobre las características de objetos que surgen de procesos infinitos en estados actuales.

La descomposición genética que se presentó en este artículo analiza la construcción de un infinito no numerable. En investigaciones previas solo se había estudiado la construcción de procesos iterativos infinitos, esto es, sucesiones definidas del conjunto de los números naturales en otro conjunto (Brown et al., 2010; Roa-Fuentes y Oktaç, 2014; Villabona y Roa-Fuentes, 2016).

La Totalidad como una estructura en la teoría APOE puede generar un impacto en la forma en la que la teoría es usada para caracterizar las construcciones cognitivas de los individuos cuando aprenden matemáticas. Las evidencias de Totalidad como una estructura en la construcción de diversos conceptos y nociones en matemáticas representan un tema importante de investigación en matemática educativa; este documento puede ser un apoyo para quienes se tracen dicho objetivo.

\section{AGRADECIMIENTOS}

A los estudiantes y profesores que contribuyeron con sus reflexiones en el desarrollo de este proyecto.

Al Programa de Movilidad de la Vicerrectoría de Investigación y Extensión de la Universidad Industrial de Santander (VIE - UIS, Colombia).

Al Proyecto C-2018-01 de la Vicerrectoría de Investigación y Extensión de la Universidad Industrial de Santander (VIE - UIS, Colombia). 


\section{REFERENCIAS}

Arnon, I., Dubinsky, E., Cottrill, J., Oktaç, A., Roa-Fuentes, S., Trigueros, M. y Weller, K. (2014). APOS theory - a framework for research and curriculum development in mathematics education. New York: Springer. http://dx.doi.org/10.1007/978-1-4614-7966-6

Artigue, M. (1995). La enseñanza de los principios del Cálculo: problemas epistemológicos, cognitivos y didácticos. En P. Gómez (Ed.), Ingeniería Didáctica en Educación Matemática. Un esquema para la investigación y la innovación en la enseñanza y el aprendizaje de las matemáticas (pp. 97-140). Bogotá, Colombia: Grupo Editorial Iberoamérica.

Asiala, M., Brown, A., DeVries, D., Dubinsky, E., Mathews, D. y Thomas, K. (1996). A framework for research and curriculum development in undergraduate mathematics education. En J. Kaput, A. H. Schoenfeld y E. Dubinsky (Eds.), Research in Collegiate Mathematics Education II (pp. 1-32). American Mathematical Society. CBMS Issues in Mathematics Education, 6. http://dx.doi.org/10.1090/cbmath/006/01

Breidenbach, D., Dubinsky, E., Hawks, J. y Nichols, D. (1992). Development of the process conception of function. Educational Studies in Mathematics, 23(3), 247-285. https://doi.org/10.1007/bf02309532

Brown, A., McDonald, M. y Weller, K. (2010). Step by step: Infinite iterative processes and actual infinity. En F. Hitt, D. Holton y P. W. Thompson (Eds.), Research in Collegiate Mathematics Education VII (pp. 115-141). American Mathematical Society. CBMS Issues in Mathematics Education, 16. http://dx.doi.org/10.1090/cbmath/016/05

Brousseau, G. (2002). Theory of didactical situations in mathematics. Nueva York: Kluwer Academic Publishers.

Cornu, B. (1992). Limits. En D. Tall (Ed.), Advanced mathematical thinking (pp. 153-166). Dordrecht, Países Bajos: Kluwer Academic.

Cottrill, J., Dubinsky, E., Nichols, D., Schwingendorf, K., Thomas, K. y Vidakovic, D. (1996). Understanding the limit concept: Beginning with a coordinated process scheme. Journal of Mathematical Behavior, 15(2), 167-192.

https://doi.org/10.1016/S0732-3123(96)90015-2

Dreyfus, T. y Tsamir, P. (2004). Ben's consolidation of knowledge structures about infinite sets. Journal of Mathematical Behavior, 23, 271-300.

http://dx.doi.org/10.1016/j.jmathb.2004.06.002

Dubinsky, E., Weller, K., McDonald, M. y Brown, A. (2005a). Some historical issues and paradoxes regarding the concept of infinity: An APOS-Based analysis: Part 1. Educational Studies in Mathematics, 58, 335-359.

http://dx.doi.org/10.1007/s10649-005-2531-z

Dubinsky, E., Weller, K., McDonald, M. y Brown, A. (2005b). Some historical issues and paradoxes regarding the concept of infinity: An APOS analysis: Part 2. Educational Studies in Mathematics, 60, 253-266. http://dx.doi.org/10.1007/s10649-005-0473-0

Dubinsky, E., Weller, K., Stenger, K. y Vidakovic, D. (2008). Infinite iterative processes: The Tennis Ball Problem. European Journal of Pure and Applied Mathematics, 1(1), 99-121.

Dubinsky, E., Weller, K. y Arnon, I. (2013). Preservice teachers' understanding of the relation between a fraction or integer and its decimal expansion: The Case of $0.999 \ldots$ and 1 . Canadian Journal of Science, Mathematics, and Technology Education, 13(3), 232-258. http://dx.doi.org/10.1080/14926156.2013.816389 
Fischbein, E. (1987). Intuition in Science and Mathematics. Dordrecht: Reidel Publ. https://doi.org/10.2307/3619981

Fischbein, E. (2001). Tacit models and infinity. Educational Studies in Mathematics, 23(48), 309-329. https://doi.org/10.1023/a:1016088708705

Hauchart, C. y Rouche, N. (1987). Apprivoiser L'infini: Un Enseignement des Débuts de L'analyse. Lovaina: CIACO.

McDonald, M., Mathews, D. y Strobel, K. (2000). Understanding sequences: A tale of two objects. Research in Collegiate Mathematics Education IV. CBMS issues in Mathematics Education (vol. 8, pp. 77-102). Providence, RI: American Mathematical Society.

Mena-Lorca, A., Mena-Lorca, J., Montoya-Delgadillo, E., Morales, A. y Parraguez, M. (2015). El obstáculo epistemológico del infinito actual: persistencia, resistencia y categorías de análisis. Revista Latinoamericana de Investigación en Matemática Educativa, 18(3), 329-358. http://dx.doi.org/10.12802/relime.13.1832

Moreno, L. y Waldegg, G. (1991). The conceptual evolution of actual mathematical infinity. Educational Studies in Mathematics, 22, 211-231. https://doi.org/10.1007/bf00368339

Oktaç, A. (2019). Mental constructions in linear algebra. ZDM Mathematics Education, 51(7), 10431054. https://doi.org/10.1007/s11858-019-01037-9

Orts, A., Llinares, S. y Boigues, F. J. (2016). Elementos para una Descomposición Genética del concepto de recta tangente. Avances de Investigación en Educación Matemática, 10, 111-134.

Piaget, J. y García, R. (1982). Psicogénesis e Historia de la Ciencia. México: Siglo XXI Editores.

Roa-Fuentes, S. y Oktaç, A. (2014). El infinito potencial y actual: descripción de caminos cognitivos para su construcción en un contexto de paradojas. Educación Matemática, 26(1), 73-101.

Sacristán, A. I. (1991). Los obstáculos de la intuición en el aprendizaje de procesos infinitos. Educación Matemática, 3(1), 5-18.

Sierpinska, A. (1987). Humanities students and epistemological obstacles related to limits. Educational Studies in Mathematics, 18, 371-397. http://dx.doi.org/10.1007/BF00240986

Villabona, D. y Roa-Fuentes, S. (2016). Procesos iterativos infinitos y objetos trascendentes: un modelo de construcción del infinito matemático desde la teoría APOE. Educación Matemática, 28(2), 119-150.

Weller, K., Brown, A., Dubinsky, E., McDonald, M. y Stenger, C. (2004). Intimations of Infinity. Notices of the AMS, 51(7), 741-750.

Wijeratne, C. y Zazkis, R (2016). Exploring conceptions of infinity via super-tasks: A case of Thomson's Lamp and Green Alien. Journal of Mathematical Behavior, 42, 127-134.

https://doi.org/10.1016/j.jmathb.2016.04.001 


\title{
Dynamic and Static Conceptions of Infinity: Continuous Processes and their Totalities
}

\author{
Diana Villabona \\ Departamento de Matemática Educativa. \\ Cinvestav - IPN, Ciudad de México, México. \\ diana.villabona@gmail.com \\ Solange Roa Fuentes \\ Escuela de Matemáticas, Facultad de Ciencias, Universidad Industrial de Santander, Bucaramanga, Colombia. \\ sroa@matematicas.uis.edu.co \\ Asuman Oktaç \\ Departamento de Matemática Educativa, Cinvestav - IPN, Ciudad de México, México. \\ oktac@cinvestav.mx
}

Potential and actual aspects of infinity and the tension between them have been the subject of studies in mathematics education that are carried out from epistemological, cognitive, as well as didactic points of view. Initial studies performed from the perspective of APOS theory associated the conceptions of potential and actual infinities to the structures of Process and Object, respectively. More recently a possible new structure called Totality has been identified in the context of repeating decimals.

Our objective in this article is twofold: on the one hand, to offer evidence for the possible structure Totality in a context involving infinity but different from the one that gave rise to the first observations; and on the other hand, to make progress and contribute to the theoretical reflections about the mental structures that constitute APOS theory.

In this article a genetic decomposition about mathematical infinity that contemplates Totality is presented. This structure is characterized by considering the concept of infinity as a whole, but not being able to apply Actions on it. The viability of the theoretical analysis is evidenced by empirical data obtained through interviews while participants worked on specially designed activities. Apart from providing evidence for a conception of Totality, the mechanisms that allow transiting from a Process to a Totality conception, as well from a Totality to an Object conception are discussed.

The design of the mathematical situation that involves limits in the context of a tangent line to a curve allowed differentiation between Totality and Object conceptions and the characterization of the mechanism that we call "completeness", which allows going from a Process to a Totality conception. This way, for the first time evidence is provided for the possible structure of Totality in a context involving a continuous process. 
